Case report

\title{
Bilateral axillary lymphadenopathy: A rare manifestation of Plasmodium falciparum malaria
}

\author{
B.Nisahan ${ }^{1}$, T.Kumanan ${ }^{1}$, G.Selvaratnam ${ }^{1}$ \\ Sri Lankan Journal of Infectious Diseases 2014 Vol.4(2):118-120 \\ DOI: http://dx.doi.org/10.4038/sljid.v4i2.7340
}

\begin{abstract}
Malaria can present with a variety of signs and symptoms. Periodic febrile paroxysms associated with rigors and sweating is classical of malaria. Malaria presenting with lymphadenopathy is an extremely rare manifestation. Lymphadenopathy is considered as one of the negative findings in diagnosing malaria. We describe a case of a 25year old male who presented with fever, confusion and bilateral axillary lymphadenopathy, diagnosed to have Plasmodium falciparum malaria. He was successfully treated with antimalarial drugs and the lymphadenopathy subsequently regressed.
\end{abstract}

Key words: Falciparum malaria, axillary lymphadenopathy, atypical manifestation

\section{Introduction}

Malaria causes significant morbidity and mortality globally. The classical presentation of malaria is with paroxysms of fever associated with rigors and sweating, which is however seen only in $50-70 \%$ of patients with malaria. ${ }^{1}$ The usual manifestations of malaria are febrile paroxysms, rigors, sweats, headache, back pain, abdominal pain, vomiting, diarrhoea, pallor and jaundice. Atypical presentation of malaria is common in endemic areas which lead to a diagnostic dilemma. Atypical manifestation include cough, jaundice, bradycardia, postural hypotension, migraine, urticarial rash, pancytopenia, cerebral involvement, cerebellar ataxia, visual loss, haemophagocytic syndrome, haemolytic uraemic syndrome, acute respiratory distress syndrome, myocarditis and splenic rupture. ${ }^{1}$ Malaria presenting with lymphadenopathy is extremely rare.

\section{Case report}

A 25year old previously healthy male presented with a 4 day history of fever with chills and rigors associated with vomiting, headache and blurring of vision. He also had obtundation. No respiratory or bowel symptoms were noted on presentation. He had reduced urine output since the day of admission. He had returned from Ghana, an African country, one week before admission after a stay of 3 months. He was not on malarial prophylaxis. The patient was drowsy

${ }^{1}$ University Medical Unit, Teaching hospital Jaffna, Sri Lanka

Address for correspondence: Dr. B Nisahan, 51/4, Potpathy road,East Kokuvil, Jaffna

Telephone No:+ 94777290755 Email-nisahan2004@yahoo.com

Received 1 September 2014 and revised version accepted 15 September 2014 
on admission. Cardiovascular, respiratory and abdominal examination was unremarkable. Bilateral small multiple axillary lymph node enlargement was noted. The largest lymph node was $2 \times 2 \mathrm{~cm}$ in size and the smallest $1 \times 1 \mathrm{~cm}$. The lymph nodes were soft to firm in consistency and mild tenderness was noted. Cranial nerve examination was unremarkable. There were no focal neurological signs, papilloedema or neck stiffness noted on clinical examination.

Complete blood count showed severe thrombocytopenia of $40,000 / \mathrm{mm}^{3}$. Renal function tests revealed a blood urea of $44 \mathrm{mmol} / \mathrm{L}$ and serum creatinine of $170 \mu \mathrm{mol} / \mathrm{L}$. Serum electrolytes, liver function tests, urine analysis, coagulation screen, cerebrospinal fluid analysis and lactate dehydrogenase level were all normal. Urine culture and blood culture were negative. Erythrocyte sedimentation rate was $60 \mathrm{~mm} / \mathrm{h}$ and there was no ultrasonic evidence of organomegaly or abdominal lymphadenopathy.

Blood film examination showed reactive leukocytes with severe thrombocytopenia and thick peripheral blood smear for malaria parasites showed Plasmodium falciparum trophozoites which was confirmed by the blood picture. The level of parasitaemia was 273 per microliter blood. Leptospira antigen and dengue antibodies were negative. Fine needle aspiration cytology of the axillary lymph node showed reactive hyperplasia.

During the hospital stay his renal function continued to deteriorate and he subsequently underwent repeated haemodialysis. He was successfully treated with oral artemetherlumefantrine combination and primaquine. His renal function returned to normal and the lymphadenopathy regressed upon recovery.

\section{Discussion}

Malaria remains one of the leading causes of morbidity and mortality. Among plasmodium species, Plasmodium falciparum is responsible for severe and fatal malaria. The symptoms and signs of malaria can be wide and none of them are diagnostic. The classical paroxysms may not be seen in many patients. In endemic areas, malaria can present with unusual features due to development of immunity, increasing resistance to antimalarial drugs and the indiscriminate use of antimalarial drugs. ${ }^{2}$

Malaria should be considered in the diagnosis of any acute febrile illness unless excluded by lack of exposure and repeated negative examination of blood smear. Absence of lymphadenopathy, rash (except herpes simplex cold sore) and focal signs are considered useful negative findings in the clinical diagnosis of malaria.

Lymphadenopathy can be demonstrated in a variety of diseases. Malaria causing abdominal lymphadenopathy and cervical lymphadenopathy has been reported previously.,4 This case illustrates that malaria cannot safely be excluded if there is lymphadenopathy, even though it is considered an important negative finding. Other causes of lymphadenopathy should be vigilantly looked for before making a clinical diagnosis of malaria.

This case illustrates the importance for clinicians to always consider the travel history and prevalent infective causes and their atypical presentations to avoid diagnostic delay. 
Declaration of conflicting interests

None declared

Funding

This case report received no specific grants from any funding agency in the public, commercial or not-for-profit sectors.

\section{References}

1. Zaki SA, Shanbag P. Atypical manifestations of malaria. Res Rep Trop Med 2011; 2:9-22 doi: 10.2147/RRTM.S13431

2. Singh UK, Kumar R, Sharma VK. Increased urinary frequency as a presentation of Plasmodium falciparum malaria. Pediatr Infect Dis J. 1994; 13(11):1024. doi: http;//dx.doi.org/10.1097/00006454-199411000-0002

3. Sood A, Midha V. Abdominal lymphadenopathy in malaria. Clin Infect Dis. 1999; 28:400-1. doi: http://dx.doi.org/10.1086/517198

4 Ger C, Kumar N, Malhotra N. Cervical lymphadenopathy: a rare presentation of malaria. J Assoc. Physicians India. 2010; 58:264. No doi 